\title{
Fusing Tissue Engineering and Systems Biology Toward Fulfilling Their Promise
}

\author{
Benjamin D. Cosgrove, ${ }^{1,2,3}$ Linda G. Griffith, ${ }^{1,3}$ and Douglas A. Lauffenburger ${ }^{1,2,3}$ \\ ${ }^{1}$ Department of Biological Engineering, Massachusetts Institute of Technology, Cambridge, MA 02139, USA; ${ }^{2}$ Cell Decision \\ Processes Center, Massachusetts Institute of Technology, Cambridge, MA 02139, USA; and ${ }^{3}$ Biotechnology Process Engineering \\ Center, Massachusetts Institute of Technology, Cambridge, MA 02139, USA
}

(Received 22 January 2008; accepted 7 February 2008; published online 11 March 2008)

\begin{abstract}
Tissue engineering has progressed to enable development of engineered 3D in vitro tissue models that can recapitulate in vivo cellular physiologies through the control of an expansive variety of microenvironmental design features, including mechanical, extracellular matrix, and soluble stimulatory cues. Microenviromental cues stimulate cells through a system of interconnected molecular regulatory pathways that govern cellular behaviors within engineered tissue models. Detailed understanding of how cell signaling pathways process these microenvironmental stimuli to govern their behaviors will require application of systems-level biological approaches. To date, the experimental and modeling approaches at the heart of systems biology have largely been examined in relatively simple experimental contexts that are readily and repeatedly addressable, such as mammalian cell lines in 2D culture. To enhance the prospects for systems biology to bring about insight into the complex cellular processes underlying human physiology and disease, and to identify and validate candidate therapeutic approaches, it needs to be advanced into improved experimental contexts that more effectively represent the complexity and functionality of native tissues. Herein, we discuss how systems biology can be adapted to the experimental constraints and interests of tissue engineering, and suggest how systems biology could aid in designing and investigating engineered tissue models for the study of human physiology and pathophysiology.
\end{abstract}

Keywords-Tissue arrays, Multivariate measurement, Partial least-squares regression, Decision trees, Bayesian networks.

\section{INTRODUCTION}

Tissue engineering is a biotechnology centered on developing materials, scaffolds, or devices that provide biochemical and biophysical cues to facilitate cell survival, proliferation, differentiation, and organization into functional three-dimensional (3D) tissues. ${ }^{19}$ The field of tissue engineering began decades ago with a

Address correspondence to Douglas A. Lauffenburger, Department of Biological Engineering, Massachusetts Institute of Technology, Cambridge, MA 02139, USA. Electronic mail: lauffen@mit.edu focus on in vivo therapeutic constructs, but has progressed to now include a substantial emphasis on the likely greater impact of providing more effective experimental systems for studying complex human tissue physiology and pathophysiology in vitro. ${ }^{20}$ This direction emerged in part because animal models fail to capture many crucial facets of human physiology, notably in the areas of tissue-specific transcriptional regulation, ${ }^{51}$ drug-induced liver toxicity, ${ }^{60}$ pathogenic infection, host immune responses, and cancer. ${ }^{56,66}$ Further, though human cells cultured in standard formats can be adapted to high-throughput assays, most scalable cell cultures lack physiologically relevant microenvironmental stimuli of native tissues. Engineered tissues built with human cells are thus being developed for a range of application areas, including hepatic drug metabolism and toxicity, ${ }^{37,45,60}$ mammary gland morphogenesis and oncogenesis, ${ }^{8,53}$ lymphoid tissue neogenesis, ${ }^{26,63}$ and stem cell differentiation, ${ }^{12}$ and offer promise for scaling to the data collection demands of high-throughput screening and systems biology.

In tissues, individual cells are stimulated by a diverse set of microenvironmental cues that arise from adhesion to extracellular matrix (ECM) components, mechanical forces, and soluble signaling factors from adjacent and distant cells. ${ }^{20}$ Together, these cues activate a system of cell signaling pathways whose integrated operation regulates cell behavioral phenotypes. ${ }^{16}$ Resultant cell behaviors are dependent not only on which microenvironmental cues are present, but also on their quantitative amounts, spatial arrangements, and temporal sequences. A central challenge in tissue engineering is to elicit and maintain desired cell behaviors through externally-applied and -induced chemical signals and mechanical forces in a predictable fashion. A tremendous diversity of tools - including biomaterials, bioreactors, and microfabricated devices - have been developed to manipulate tissue microenvironments. ${ }^{20,36}$ 
Design principles for deploying these tools are likewise emerging, but they are primarily aimed at relating the magnitude of select external cues directly to cell phenotypic behaviors through quantitative analysis of molecular diffusion, convection, reaction, binding, and consumption. $^{20,22}$ In increasingly complex engineered microenvironments, detailed understanding of how the multitude of cell signaling pathways process various microenvironmental cues to govern their behaviors will require application of systems-level approaches. To this end, systems biology offers a powerful new tool for the design and analysis of engineered tissues.

Systems biology seeks a deep quantitative understanding of complex biological processes through the integration of multivariate molecular-level measurement and modeling approaches, and thus differs from proteomic and genomic efforts that aim to catalogue a broad listing of biological components and their functions. ${ }^{1,25}$ Advances in high-throughput and multiplex techniques for quantifying the abundances and activities of the molecular components involved in gene expression, ${ }^{38}$ metabolism, ${ }^{50}$ and signal transduction $^{1}$ make it feasible to collect large data sets of diverse cellular processes. Large-scale quantitative studies of complex biomolecular processes are difficult to interpret by inspection and intuition alone. Computational modeling allows simplification of large-scale biological data sets and can suggest mechanistic insights and enable quantitative predictions of cellular processes. ${ }^{25,31}$

Our review here focuses on molecular-level models that attempt to relate microenvironment stimuli to intracellular signal transduction pathways and their regulation of cellular behavioral responses. For discussion of systems-level modeling of transcription and metabolism, readers are referred to other reviews. ${ }^{33,50}$ Although some promising systems-level studies ${ }^{5,12,39,62}$ have been examined in contexts that capture some of the physiological complexity of native tissues, systemslevel cell signaling-response measurements and models, to date, have been largely implemented in simple, "prototypical" experimental contexts. In this review, we discuss how systems biology might be more fully integrated into the design and analysis of engineered tissues. A schematic illustration of the key facets of our address is provided in Fig. 1.

\section{SYSTEM-LEVEL MEASUREMENT OF CELL SIGNALING AND BEHAVIORAL PHENOTYPES}

In systems biology, the collection of experimental data sets is organized and conducted with regard to intended modeling efforts and practical limitations. Systems models largely require dynamic, highly

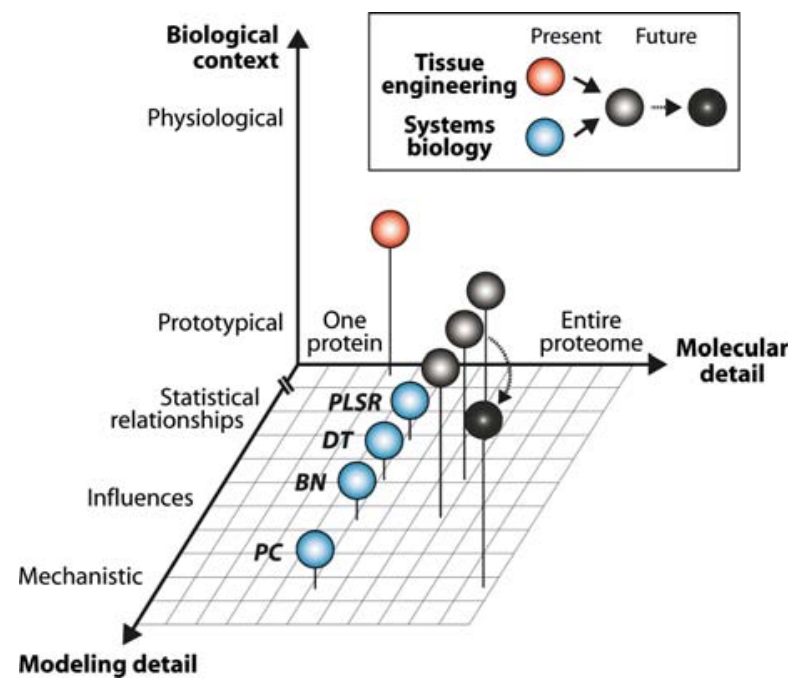

FIGURE 1. The scope of measurement and modeling in systems biology and tissue engineering. The present and future implementation of systems biology and tissue engineering are depicted with respect to: biological context (ranging from prototypical cell lines to engineered and native tissues to whole organisms); modeling detail (ranging from statistical models to influence models to mechanistic models); and molecular detail (ranging from a single protein to the entire proteome). To date, tissue engineering has been focused on the recapitulation of the physiological complexity of native tissues but has not been studied in great molecular detail nor has been fused with systems-level computational modeling efforts. In contrast, systems-level computational models, such as partial least-squares regression (PLSR), decision trees (DT), Bayesian networks (BN), and physicochemical (PC) models, can interpret a variety of molecular relationships governing cellular behavior but have been studied largely in a prototypical biological context (e.g., cell lines). Future fusion of tissue engineering and system biology will require adaption of tissue engineering to the measurement demands systems-level computational models. Considering these demands, PLSR, DT, and Bayesian models provide attractive candidates for analysis of cell behaviors within engineered tissues in the near future. More substantial progress in tissue engineering and multivariate measurement technologies will be required for implementation of models with more mechanistic and molecular detail.

multivariate, and quantitative data of protein activities and cell behavioral phenotypes collected in specific cell types subjected to defined, and often diverse, stimuli. A wide variety of high-throughput and multiplex experimental techniques are utilized to collect data sets for systems models. These include mass spectrometry, ${ }^{67}$ kinase activity assays, ${ }^{28,59}$ immunoblotting, ${ }^{14}$ 'in-cell westerns, ${ }^{41}$ bead-based arrays ${ }^{48}$ protein microarrays, ${ }^{40,58}$ and multicolor flow ${ }^{54,57}$ and image ${ }^{11,46}$ cytometry.

In selecting appropriate measurement techniques, one often considers the identity and number of simultaneously measurable signals (whether assays can be 'multiplexed'), the amount of sample required per assay, and whether single-cell or population-level behavior is measured. ${ }^{1}$ Mass spectrometry can be used to quantify the relative abundance of hundreds of 
regulatory protein phosphorylation sites across multiple biological samples, but requires $10^{5}-10^{7}$ cells per sample due to the fractionation required to detect low abundance peptides in a complex cellular lysate. ${ }^{71}$ Kinase activity assays directly measure the enzymatic activity of kinases to phosphorylate substrates via radiochemical $^{28}$ or fluorescence ${ }^{59}$ readouts and are multiplexible but require $\sim 10^{5}$ cells per kinase assayed. 'In-cell westerns' are a form of immunofluorescence microscopy that measures protein levels or states in fixed cells still adherent to culture surfaces; ${ }^{41}$ this method is not currently multiplexible but requires only $10^{4}-10^{5}$ cells per protein assayed. Bead-based arrays utilize flow cytometric quantification of bead-conjugated, fluorescently labeled antibody sandwich assays. ${ }^{48}$ A number of commercial vendors provide well-validated, highly multiplexible reagents for phosphoprotein quantification in a cell lysate using the Luminex platform. In practical applications, 10-20 phosphoproteins can be quantified in a lysate from $10^{4}-10^{5}$ cells. Protein microarrays allow detection of binding avidities of cellular proteins to hundreds of printed protein and/or substrate features and can be conducted with a lysate from $10^{4}$ to $10^{5}$ cells. ${ }^{58} \mathrm{Mul}-$ ticolor flow and image cytometry can assess phosphoprotein levels and cellular phenotypes (such as proliferation, apoptosis, and migration) at a single-cell level. Using well-validated antibodies and stains, multiplexing $\sim 10$ and $\sim 4$ parallel measurements are typical current practical upper limits for flow ${ }^{54}$ and image ${ }^{46}$ cytometry, respectively, but informative data sets can be collected in as few as $10^{2}-10^{3}$ cells per condition.

As no individual measurement technique can capture the full diversity of protein signals important to the operation of cell signaling networks, systems biology models of cell signaling and responses are increasingly relying on data compendia assembled from heterogeneous assay types. ${ }^{14}$ Assembly of such data compendia requires careful consideration to data fusion, normalization, and scaling when applied to quantitative models. Readers are referred elsewhere ${ }^{1,14}$ for discussions of data compendia assembly and validation.

\section{DATA-DRIVEN MODELING OF CELL SIGNALING AND BEHAVIORAL PHENOTYPES}

A wide spectrum of computational modeling approaches for studying cell signaling and its regulation of behavioral phenotypes is available. ${ }^{30}$ Because the mechanisms connecting disparate cell signaling pathways to each other and to integrated cell behaviors are largely unknown, approaches such as differential equation-based physicochemical models ${ }^{3}$ that require substantial mechanistic knowledge are currently limited in their practical applicability for analyzing relationships between regulatory network activities and downstream cell phenotypic behavior. Accordingly, we focus here on a set of more abstract modeling approaches more suitable for characterizing the operational relationships, influences, and logic of cell signaling networks as they relate to cell functions: partial least-squares regression (PLSR), decision trees, and Bayesian network inference.

\section{Partial Least-Squares Regression}

Partial least-squares regression (PLSR) models are based on the hypothesis that, across multiple treatment conditions, cell behavioral phenotypes ('responses') are inherently dependent on quantitative combinations of a subset of measurable activities or states of key signaling molecules ('signals'). ${ }^{27,31}$ Measured signaling variables (including both protein activities at multiple time points and extracted time-dependent metrics) and measured cell behaviors are cast into two separate data matrices: an independent block of signaling variables $(X)$ and a dependent block of response variables $(Y)$, both arrayed across multiple cellular conditions. Since the number of cellular conditions measured often is exceeded by the number of signaling variables, PLSR is necessary to calculate a unique regression solution to the hypothesized relationship $Y=f(X)$. PLSR identifies a linear solution to the signaling-response relationship within a reduced-dimensionality data space defined by a set of orthogonal principal components. ${ }^{15}$ (It should be noted that nonlinear relationships can be readily modeled through inclusion of nonlinear combinations of variables and/or variable transformations.) The calculation of principal components-based regression weights is biased towards those signaling variables that most strongly correlate with the responses and to optimize prediction accuracy of the responses in cross-validation.

PLSR models can be used to elucidate an integrative model of network operation that can identify key combinations of signaling activities governing measured cell behaviors. Moreover, they can be used to quantitatively and accurately predict responses of cells to additional treatments, such as pharmacologic perturbations, a priori using newly measured and/or estimated signaling data. To generate a model capable of accurate a priori predictions, the conditions used to train a PLSR model need to strongly and differently activate the breadth of measured cell signaling activities and behaviors. ${ }^{14,47}$ PLSR models have been generated using cell signaling and response data from a number of the aforementioned measurement techniques, and have been successful at interpreting and 
predicting cell signaling-response relationships in varied contexts such as ECM-regulated embryonic stem cell self-renewal and differentiation, ${ }^{55}$ cytokine- and pathogen-induced epithelial cell apoptosis-survival, ${ }^{14,27,47}$ receptor agonist-induced T-cell and B-cell cytokine release, ${ }^{34,43}$ and growth factor-induced mammary epithelial cell proliferation and migration. ${ }^{44}$

\section{Decision Trees}

Decision tree (DT) models can be useful as networkfunction models to interpret and predict cell signalresponse relationships in terms of logical combinations of multiple signal activity levels. Decision tree models are generated from a learning algorithm that approximates a cellular output by constructing a "tree" where the "branches" classify logical combinations of signals based on their measured or estimated levels, and the "leaves" at the end of the branches predict the cellular output. The idea behind DT modeling is to recursively split signal-response data into successively smaller branches in order to end up with a tree in which signal combination branches are obtained that can classify response behavior as correctly as possible. A decision tree model is usually obtained via a two-step process. $^{4,21}$ The first step, tree growing, is done until all response observations are classified correctly. The second step, tree pruning, is done in order to avoid over-fitting. In generally, decision tree models have several properties that are appealing in biomedical research ${ }^{4}$ : (a) they can be effectively applied to broad classes of data, in particular to discrete, continuous or mixed data; (b) they are capable of good prediction accuracy for highly nonlinear prediction problems; (c) their prediction rules are easy to visualize and interpret; and (d) they are very robust against outliers. Additionally, a DT model provides quantitative predictions to guide interventions, such as using pharmacologic inhibitors, even when such interventions only partially diminish the signal. Moreover, the DT approach facilitates incorporation of nonlinear behavior more readily through its alternative inclusions of qualitatively diverse model branches characterizing different logical relationships among the signaling nodes. Successful decision tree models have been developed for growth factor- and ECM-induced fibroblast migration ${ }^{21,35}$ and Fas-mediated $\mathrm{T}$ lymphoma apoptosis. $^{23}$

\section{Bayesian Networks}

Bayesian networks offer a modeling technique for identifying causal relationships among multiple signals as well as from signals to responses. Bayesian network models illustrate the interactions of pathway components in the form of influence diagrams that can contain both direct molecular interactions (e.g., kinase-substrate phosphorylation) and indirect influences that proceed through unobserved intermediates. ${ }^{57}$ Models are inferred through probabilistic calculations of covariations in activities of multiple signaling species, usually sparsely distributed across different signaling pathways, collected from single cells $^{57}$ or populations of cells ${ }^{68}$ exposed to diverse combinations of extracellular stimuli and genetic an$\mathrm{d} /$ or pharmacologic pathway interventions, with effective inference requiring many observations. As such, flow cytometry data containing simultaneous measurement of multiple phosphoproteins of thousands of individual cells under a variety of treatment conditions are well-suited for Bayesian inference and have resulted in accurately inferred influence networks. ${ }^{57}$ In comparison, Bayesian models based on population-based multivariate signaling data ${ }^{57,68}$ require more diverse treatments and have reduced network inference accuracy, in part due to the obfuscation of informative heterogeneity at the single-cell level. Inferred influence networks can suggest novel signal transduction hypotheses ${ }^{57}$ but also could be useful in identifying context-specific signaling network structures. This kind of model can also be used to make predictions about how interventions in the network influence downstream cell phenotypic behaviors, as demonstrated for the example of embryonic stem cell self-renewal and differentiation responses to combinations of cytokine and extracellular matrix cues. ${ }^{68}$ We should emphasize that Bayesian network modeling efforts to date have been restricted to static models, but the feedback loops inherent in cell signaling networks will likely often require dynamic Bayesian network models adapted to handle cyclic connectivities. ${ }^{6}$

\section{FUSING TISSUE ENGINEERING AND MULTIVARIATE MEASUREMENT METHODS}

Systems-level models require experimental data of activities of multiple cell signaling molecules and behavioral phenotypes across a diverse combination of treatments, perturbations, and time points. Accordingly, they require cell culture systems that are both addressable in a high-throughput manner and amenable to multivariate measurement methods. Recent advances offer promise for parallelizing the culture of microenvironmentally complex engineered tissues (in so-called 'multicellular arrays' or 'tissue arrays') sufficient to meet the data demands of systems biology. Culture arrays have risen out of the desire to screen phenotypes of multicellular structures in a high-throughput manner, and have been applied to drug discovery ${ }^{64}$ and toxicology ${ }^{45}$ 
and combinatorial approaches to directing and maintaining cell differentiation. ${ }^{13,18,62,65}$ Such combinatorial screens of cell differentiation have provided unexpected insights into relationships between multiple microenvironmental cues ${ }^{13}$ (e.g., printed ECM ligands) or intracellular signals ${ }^{55,62}$ and the maintenance of differentiated phenotypes through multiparameter analyses. (Note that we refer to culture arrays with $\sim 10^{2}-10^{3}$ cells per spot or well as 'tissue microarrays' and those with $\sim 10^{5}-10^{6}$ cells per sample well as 'multiwell-format tissue arrays'.)

We wish to emphasize that systems biology models to date have focused on responses to "acute," bolus administration of cytokines and growth factors and thus have inferred substantial predictive significance to the transient signals and derived time course metrics immediately following receptor activation. ${ }^{14}$ However, in tissue engineered constructs as in physiological contexts, most cell behaviors more likely are governed by longer time-scale stimuli, under more "chronic," quasi-steady-state conditions. There might indeed be significant differences in relationships between cues, signals, and responses for chronic as opposed to acute cue treatment, as in a recent report of important pathophysiological differences in 2D mammary epithelial migration by autocrine ligands or bolus stimulation from exogenously-added ligand. ${ }^{32}$

\section{Tissue Microarrays}

Efforts in developing tissue microarrays have been focused on validating consistency of cellular phenotypes and assays such as drug-induced hepatocyte toxicity, ${ }^{45,52}$ stem cell differentiation, ${ }^{18}$ and epithelial tissue organization, ${ }^{2,61}$ and have not been used to systematically examine cue- or signalresponse relationships. A tissue microarray system well-suited for systems biology approaches is that of mammary epithelial cells in microprinted Matrigel cultures. ${ }^{61}$ Matrigel culture of mammary epithelial cells fosters the establishment of 3D multicellular structures that resemble in vivo mammary gland acini, with the development epithelial cell structures that resemble either normal or cancerous mammary acini depending on a well-documented variety of both microenvironmental cues (such as matrix mechanical stiffness and composition and growth factor stimulation) and oncogenic perturbations. $^{7,8,53,69}$ As of yet, tissue arrays have not been adapted to allow inclusion of ECM components with a combinatorial complexity matching that used in the $2 \mathrm{D}$ microprinting methods ${ }^{13}$ which will be necessary for systematic investigation of cellular cue-signal-response relationships.
Although tissue microarrays could be scaled to be amenable to lysate-based measurements (requiring $\sim 10^{5}$ cells), they are nonetheless appropriate for systems biology approaches, such as Bayesian network inference, that are based on data from single cells or small populations of cells examined over a multitude of conditions. Critical will be the adaption of singlecell multivariate measurement techniques of flow and image cytometry for use in 3D engineered tissues. Multicolor flow cytometry for quantifying multiple phosphoproteins has been carefully validated ${ }^{54}$ and implemented $^{42,57}$ for non-adherent cells but not adherent cells. Multicolor flow cytometry in adherent cells, especially from intact 3D tissues, is a significant challenge as single-cell isolation methods can elicit stress-related signaling themselves and thus disturb signaling network states. Further, prolonged isolation requirements could restrict the ability to measure the highly-informative phosphorylation events that follow within minutes of cell stimulation. In contrast, image cytometry does not require isolation and permits for immediate cell fixation, allowing for sufficient temporal resolution to measure immediate phosphorylation events. Instead, it is currently limited in its ability to include more than $\sim 4$ simultaneous single-cell measurements due technical constraints. ${ }^{46}$ Multicolor 3D imaging (so-called 'tissue cytometry' ${ }^{11}$ ) using confocal microscopy is not yet sufficiently highthroughput to capture multivariate single-cell data across hundreds of tissue samples in an automated fashion. Improvements in 3D imaging with respect to these limitations could make it a highly attractive approach for system-level, single-cell data acquisition, especially in the context of engineered tissues, such as immunological synapse arrays, ${ }^{9}$ whose cellular behaviors are dependent on microscale patterning of stimulatory cues thus necessitate spatially-resolved single-cell data.

Immunofluorescence methods, such as in-cell westerns, which are reasonably high-throughput but not strictly multiplexible, could be used to measure phosphoprotein levels or cell phenotypes in small populations of cells $\left(\sim 10^{2}-10^{3}\right)$ within tissue microarrays. In-cell western measurements of arrayed tissues over a large combination of treatment conditions, assay targets, and, likely, sample replicates could yield a data set sufficient for Bayesian models. As in-cell western measurements are not multiplexible, multivariate data sets will require assembly from multiple independent samples of cells, thus obscuring the single-cell covariations of signaling activities that are particularly informative in Bayesian models. ${ }^{57}$ Consequently, a substantial set of microenvironmental stimuli combinations ( 100) might be required for accurate Bayesian network inference. Moreover, care must be taken to 
insure that the biological heterogeneity, due to the small number of cells per sample, and measurement inaccuracy, which often arises due to the restrictive dynamic range of in-cell westerns, does not confound multivariate measurement fusion. A correlated, and promising, method called "stochastic sampling" employs laser-capture microdissection and a customized quantitative PCR method to perform multiplexed gene expression profiling in small populations of cells $(\sim 10)$ has lead to novel insights into the regulation of transcription programs controlling heterogeneous cell behaviors in 3D mammary acini cultures. ${ }^{29}$

\section{Multiwell-Format Tissue Arrays}

Multiwell-format tissue arrays $^{10,60}$ are based on 12- or 24-well culture formats and can accommodate $\sim 10^{5}-10^{6}$ cells per sample well (sufficient for lysatebased measurements) and be scaled to a moderate number of sample conditions (i.e., treatments, time points) and thus are suitable for a greater diversity of systems-level modeling approaches than tissue microarrays. A well-developed example of these multiwellformat tissue arrays that utilizes a 3D microenvironment is a perfused liver cell microreactor. ${ }^{10,60}$ Perfused microreactors foster the maintenance of in vivo-like function of primary hepatocytes ${ }^{60}$ and/or liver sinusoidal endothelial cells ${ }^{24}$ over a prolonged culture duration ( $\sim 14$ days) in a physiologically-relevant $3 \mathrm{D}$ microenvironment. And, as such, have been used examine hepatic drug metabolism and enzyme induction $^{60}$ and sinusoidal morphogenesis ${ }^{24}$ over physiologically-relevant time scales.

The larger cell numbers per sample permit lysate methods such as bead-based arrays ${ }^{48}$ and multiplexed kinase activity assays ${ }^{28}$ to be utilized in addition to multicolor flow and image cytometry (given the aforementioned improvements) to measure cell signaling activities and phenotypes in a multivariate manner. Bead-based phosphoprotein arrays allow for a greater number of measureable signaling molecules as they are benefited by an expansive set of validated, multiplexible reagents, while kinase activity assays have the advantage of measuring signal transduction activity directly, rather than inferred via phosphorylation state. Consequently, activity data from kinases, especially of those thought to be at nodes of signaling networks that integrate multiple pathways, are often found to be highly informative signals in PLSR cell signaling-response models trained on protein signaling data from heterogeneous assays. ${ }^{14,27}$ Practical limitations on cell material and knowledge of which protein signaling assays have been informative previously ${ }^{14}$ together suggest that PLSR models using multiwell-format tissue arrays might be best constructed using $\sim 2-3$ kinase assays and $\sim 10$ bead-based phosphoprotein measurements (all from a single lysate) of proteins distributed across multiple cell signaling pathways. A challenge in multivariate measurement of engineered liver cell co-cultures (usually containing hepatocytes and second liver cell type) is the correct attribution of signaling profiles to each of the multiple cell types present. Flow cytometry methods can simply employ one staining channel to a cell type marker, but lysate based methods require less straight-forward approaches such as those that rely on biomolecular mixture models ${ }^{17}$ to infer cell type-specific phosphoprotein profiles.

\section{IMPLEMENTING SYSTEMS-LEVEL MODELING TO THE DESIGN AND ANALYSIS OF ENGINEERED TISSUES}

In contrast to the fields of genomics and proteomics, a systems biology approach insists that knowledge and understanding about biology resides not in databases but instead in models - and that given the complexity of multivariate experimental measurements these models must be computational in nature. Thus, the data generated in tissue engineering contexts as discussed in the previous section will be most gainfully employed by analysis in terms of one or more of the data-driven modeling methods described earlier.

Data-driven models of dynamic, multivariate cell signaling and response data can suggest hypotheses that relate activities of signaling molecules to behavioral phenotypes and can be implemented to generate a priori predictions of responses to new signaling network profiles. Such models are most effectively constructed on the basis of training across a broad landscape of conditions, including conflicting or antagonizing cues, that stimulate a diversity of signaling network activities and cell responses that might be hypothesized to comprehend a range of physiological phenotypic behaviors..$^{27,34,44,47}$ Prediction of cell behavior can then be effectively interpolative rather than extrapolative. A priori prediction of cell phenotypes based on signaling network states could lead to the identification nonintuitive combinations to signaling activities (through computation searches, for example) that optimally produce a desired cell phenotype in an engineered tissue. Design of novel engineered tissue microenvironments that generate the optimal signaling network state could be furthermore governed by physicochemical models of receptor activation and downstream signaling, thus integrating mechanistic and data-driven model approaches ${ }^{23}$ to design features for fostering desired tissue phenotypes. Moreover, models of cell behavior within a tissue 
engineered to mimic a particular physiology or pathophysiology could yield models capable of predictions of drug efficacy and toxicity in physiologically relevant, tissue-specific contexts. In particular, such approaches could be used to select target pathways for interfering with 3D cell migration behaviors critical to cancer metastsis ${ }^{70}$ and matrix stiffness-induced mammary acinar oncogenesis. ${ }^{7,53}$ For instance, successful a priori PLSR model prediction of cell phenotypic changes due to perturbation by one or more small molecular inhibitor(s) has been verified for compounds with specified kinase targets that lie upstream of protein signal(s) contained in the model and implemented by 'computationally inhibiting' only the measured signals in a subset of the training data and then comparing the predicted results to new experiment observations. ${ }^{34,47}$ Perturbations that affect signaling networks more globally (therefore less predictably) such as growth factor receptor overexpression, ${ }^{43}$ disruption of autocrine ligand signaling, ${ }^{27}$ and RNA interference $^{43}$ have been evaluated using complete re-collection of signaling data rather than the straightforward estimation methods successful for small molecular inhibitors. When measuring multiple diverse kinds of cell phenotypic behaviors, as is desired for understanding complex tissue physiology, constructing separate submodels for the various behaviors might allow for easier interpretation of the various respective signaling-response relationships.

Decision tree and Bayesian network models can extend predictive capabilities to causality, more strongly indicating molecular mechanisms. For example, a Bayesian network model for the signaling network regulating differentiation versus self-renewal processes of mouse embryonic stem cells permitted experimentally validated prediction of effects of Raf1 independent of the canonical Raf-MEK-ERK pathway. ${ }^{68}$ Analogously, one can envision developing a similar predictive capability for signals governing lineage specification of mesenchymal stem cells cultured in complex 3D microenvironments containing varying adhesion ligands, matrix rigidities, and growth factors. ${ }^{12}$ Likewise, predicted signaling network influences could suggest microenvironmental cue and pharmacological intervention combinations for influencing osteoblast specification for implantable bone regeneration therapies. ${ }^{49}$

Moreover, Bayesian network inference approaches could ask fundamental questions about the perhaps subtle differences in signaling network structure and function in cells of varying lineages and disease states and how those networks diverge from canonical models. ${ }^{42}$ Such understanding could drive clinical therapies that are motivated by context-specific cell signaling networks. Bayesian approaches could be employed in the challenging task of investigating the crosstalk between multiple cell types in healthy or diseased tissues. By engineering tissue arrays that contain multiple interacting cell types, such as the immune system cells within a lymph node ${ }^{26}$ or the varied cells within the liver, ${ }^{24}$ and measuring both cell type-specific phosphoprotein signaling and levels of soluble or matrix-related signaling factors, Bayesian network inference models can be constructed to provide mechanistic hypotheses for complex cellular crosstalk in tissues.

\section{CONCLUDING NOTE}

The fields of tissue engineering and systems biology have each been motivated by an aspiration to generate more physiologically relevant approaches to understanding and manipulating biology, with the former focusing on improved experimental contexts while the latter has focused on enhanced experimental measurements and computational modeling techniques. It is clearly manifest at this point in time that each of these fields needs to become intimately fused with the other in order to fulfill their shared vision and promise. We hope that this article will be useful to many investigators, in academic as well as industrial research endeavors, to be encouraged to consider their integration as a high priority.

\section{ACKNOWLEDGMENTS}

The authors appreciate insights offered by Kevin Janes, Justin Pritchard, and Pamela Kreeger toward the ideas discussed herein. This effort derived primarily from funding support from the NIGMS Cell Decision Processes Center, the NIGMS Cell Migration Consortium, and Pfizer Research Technology Center.

\section{OPEN ACCESS}

This article is distributed under the terms of the Creative Commons Attribution Noncommercial License which permits any noncommercial use, distribution, and reproduction in any medium, provided the original author(s) and source are credited.

\section{REFERENCES}

\footnotetext{
${ }^{1}$ Albeck, J. G., G. MacBeath, F. M. White, P. K. Sorger, D. A. Lauffenburger, and S. Gaudet. Collecting and organizing systematic sets of protein data. Nat. Rev. Mol. Cell. Biol. 7:803-812, 2006.
} 
${ }^{2}$ Albrecht, D. R., G. H. Underhill, T. B. Wassermann, R. L. Sah, and S. N. Bhatia. Probing the role of multicellular organization in three-dimensional microenvironments. Nat. Methods 3:369-375, 2006.

${ }^{3}$ Aldridge, B. B., J. M. Burke, D. A. Lauffenburger, and P. K. Sorger. Physicochemical modelling of cell signalling pathways. Nat. Cell. Biol. 8:1195-1203, 2006.

${ }^{4}$ Breiman, L. Classification and Regression Trees. Belmont, CA: Wadsworth Intl. Group, p. 358, 1984.

${ }^{5}$ Calvert, V. S., R. Collantes, H. Elariny, A. Afendy, A. Baranova, M. Mendoza, Z. Goodman, L. A. Liotta, E. F. Petricoin, and Z. M. Younossi. A systems biology approach to the pathogenesis of obesity-related nonalcoholic fatty liver disease using reverse phase protein microarrays for multiplexed cell signaling analysis. Hepatology 46:166-172, 2007.

${ }^{6}$ David, L. A., and C. H. Wiggins. Benchmarking of dynamic Bayesian networks inferred from stochastic timeseries data. Ann. N Y Acad. Sci. 1115:90-101, 2007.

${ }^{7}$ Debnath, J., and J. S. Brugge. Modelling glandular epithelial cancers in three-dimensional cultures. Nat. Rev. Cancer 5:675-688, 2005.

${ }^{8}$ Debnath, J., K. R. Mills, N. L. Collins, M. J. Reginato, S. K. Muthuswamy, and J. S. Brugge. The role of apoptosis in creating and maintaining luminal space within normal and oncogene-expressing mammary acini. Cell 111:29-40, 2002.

${ }^{9}$ Doh, J., and D. J. Irvine. Immunological synapse arrays: patterned protein surfaces that modulate immunological synapse structure formation in T cells. Proc. Natl. Acad. Sci. USA 103:5700-5705, 2006.

${ }^{10}$ Domansky, K., W. Inman, J. Serdy, and L. Griffith. Perfused microreactors for liver tissue engineering. Conf. Proc. IEEE Eng. Med. Biol. Soc. 7:7490-7492, 2005.

${ }^{11}$ Ecker, R. C., and A. Tarnok. Cytomics goes 3D: toward tissomics. Cytometry A 65:1-3, 2005.

${ }^{12}$ Engler, A. J., S. Sen, H. L. Sweeney, and D. E. Discher. Matrix elasticity directs stem cell lineage specification. Cell 126:677-689, 2006.

${ }^{13}$ Flaim, C. J., S. Chien, and S. N. Bhatia. An extracellular matrix microarray for probing cellular differentiation. Nat. Methods 2:119-125, 2005.

${ }^{14}$ Gaudet, S., K. A. Janes, J. G. Albeck, E. A. Pace, D. A. Lauffenburger, and P. K. Sorger. A compendium of signals and responses triggered by prodeath and prosurvival cytokines. Mol. Cell. Proteomics 4:1569-1590, 2005.

${ }^{15}$ Geladi, P., and B. R. Kowalski. Partial least-squares regression: a tutorial. Anal. Chim. Acta 185:1-17, 1986.

${ }^{16}$ Gerhart, J. 1998 Warkany lecture: signaling pathways in development. Teratology 60:226-239, 1999.

${ }^{17}$ Ghosh, D. Mixture models for assessing differential expression in complex tissues using microarray data. Bioinformatics 20:1663-1669, 2004.

${ }^{18}$ Gomez-Sjoberg, R., A. A. Leyrat, D. M. Pirone, C. S. Chen, and S. R. Quake. Versatile, fully automated, microfluidic cell culture system. Anal. Chem. 79:8557-8563, 2007.

${ }^{19}$ Griffith, L. G., and G. Naughton. Tissue engineering-current challenges and expanding opportunities. Science 295:1009-1014, 2002.

${ }^{20}$ Griffith, L. G., and M. A. Swartz. Capturing complex 3D tissue physiology in vitro. Nat. Rev. Mol. Cell. Biol. 7:211-224, 2006.

${ }^{21}$ Hautaniemi, S., S. Kharait, A. Iwabu, A. Wells, and D. A. Lauffenburger. Modeling of signal-response cascades using decision tree analysis. Bioinformatics 21:2027-2035, 2005.

${ }^{22}$ Helm, C. L., M. E. Fleury, A. H. Zisch, F. Boschetti, and M. A. Swartz. Synergy between interstitial flow and VEGF directs capillary morphogenesis in vitro through a gradient amplification mechanism. Proc. Natl. Acad. Sci. USA 102:15779-15784, 2005.

${ }^{23}$ Hua, F., S. Hautaniemi, R. Yokoo, and D. A. Lauffenburger. Integrated mechanistic and data-driven modelling for multivariate analysis of signalling pathways. J. R. Soc. Interf. 3:515-526, 2006.

${ }^{24}$ Hwa, A. J., R. C. Fry, A. Sivaraman, P. T. So, L. D. Samson, D. B. Stolz, and L. G. Griffith. Rat liver sinusoidal endothelial cells survive without exogenous VEGF in 3D perfused co-cultures with hepatocytes. Faseb. J. 21:2564-2579, 2007.

${ }^{25}$ Ideker, T., L. R. Winslow, and D. A. Lauffenburger. Bioengineering and systems biology. Ann. Biomed. Eng. 34:1226-1233, 2006.

${ }^{26}$ Irvine, D. J., A. N. Stachowiak, and Y. Hori. Lymphoid tissue engineering: invoking lymphoid tissue neogenesis in immunotherapy and models of immunity. Semin. Immunol., 2007. In press, doi:10.1016/j.smim.2007.10.010.

${ }^{27}$ Janes, K. A., J. G. Albeck, S. Gaudet, P. K. Sorger, D. A. Lauffenburger, and M. B. Yaffe. A systems model of signaling identifies a molecular basis set for cytokine-induced apoptosis. Science 310:1646-1653, 2005.

${ }^{28}$ Janes, K. A., J. G. Albeck, L. X. Peng, P. K. Sorger, D. A. Lauffenburger, and M. B. Yaffe. A high-throughput quantitative multiplex kinase assay for monitoring information flow in signaling networks: application to sepsisapoptosis. Mol. Cell. Proteomics 2:463-473, 2003.

${ }^{29}$ Janes, K. A., and J. S. Brugge. Identifying matrix- and growth factor-induced transcriptional dichotomies by stochastic sampling. ASCB Annual Meeting, 2007.

${ }^{30}$ Janes, K. A., and D. A. Lauffenburger. A biological approach to computational models of proteomic networks. Curr. Opin. Chem. Biol. 10:73-80, 2006.

${ }^{31}$ Janes, K. A., and M. B. Yaffe. Data-driven modelling of signal-transduction networks. Nat. Rev. Mol. Cell. Biol. 7:820-828, 2006.

${ }^{32}$ Joslin, E. J., L. K. Opresko, A. Wells, H. S. Wiley, and D. A. Lauffenburger. EGF-receptor-mediated mammary epithelial cell migration is driven by sustained ERK signaling from autocrine stimulation. J. Cell. Sci. 120:3688-3699, 2007.

${ }^{33}$ Joyce, A. R., and B. O. Palsson. The model organism as a system: integrating 'omics' data sets. Nat. Rev. Mol. Cell. Biol. 7:198-210, 2006.

${ }^{34}$ Kemp, M. L., L. Wille, C. L. Lewis, L. B. Nicholson, and D. A. Lauffenburger. Quantitative network signal combinations downstream of TCR activation can predict IL-2 production response. J. Immunol. 178:4984-4992, 2007.

${ }^{35}$ Kharait, S., S. Hautaniemi, S. Wu, A. Iwabu, D. A. Lauffenburger, and A. Wells. Decision tree modeling predicts effects of inhibiting contractility signaling on cell motility. BMC Syst. Biol. 1:9, 2007.

${ }^{36}$ Khetani, S. R., and S. N. Bhatia. Engineering tissues for in vitro applications. Curr. Opin. Biotechnol. 17:524-531, 2006.

${ }^{37}$ Khetani, S. R., and S. N. Bhatia. Microscale culture of human liver cells for drug development. Nat. Biotechnol. 26:120-126, 2008.

${ }^{38}$ Kim, T. H., and B. Ren. Genome-wide analysis of proteinDNA interactions. Annu. Rev. Genom. Hum. Genet. 7:81102, 2006.

${ }^{39}$ Klingmuller, U., A. Bauer, S. Bohl, P. J. Nickel, K. Breitkopf, S. Dooley, S. Zellmer, C. Kern, I. Merfort, T. Sparna, J. Donauer, G. Walz, M. Geyer, C. Kreutz, M. Hermes, F. Gotschel, A. Hecht, D. Walter, L. Egger, 
K. Neubert, C. Borner, M. Brulport, W. Schormann, C. Sauer, F. Baumann, R. Preiss, S. MacNelly, P. Godoy, E. Wiercinska, L. Ciuclan, J. Edelmann, K. Zeilinger, M. Heinrich, U. M. Zanger, R. Gebhardt, T. Maiwald, R. Heinrich, J. Timmer, F. von Weizsacker, and J. G. Hengstler. Primary mouse hepatocytes for systems biology approaches: a standardized in vitro system for modelling of signal transduction pathways. Syst. Biol. (Stevenage) 153:433-447, 2006.

${ }^{40}$ Knickerbocker, T., J. R. Chen, R. Thadhani, and G. MacBeath. An integrated approach to prognosis using protein microarrays and nonparametric methods. Mol. Syst. Biol. 3:123, 2007.

${ }^{41}$ Kortum, R. L., D. L. Costanzo, J. Haferbier, S. J. Schreiner, G. L. Razidlo, M. H. Wu, D. J. Volle, T. Mori, H. Sakaue N. V. Chaika, O. V. Chaika, and R. E. Lewis. The molecular scaffold kinase suppressor of Ras 1 (KSR1) regulates adipogenesis. Mol. Cell. Biol. 25:7592-7604, 2005.

${ }^{42}$ Krutzik, P. O., J. M. Crane, M. R. Clutter, and G. P. Nolan. High-content single-cell drug screening with phosphospecific flow cytometry. Nat. Chem. Biol. 4:132-142, 2008.

${ }^{43}$ Kumar, D., R. Srikanth, H. Ahlfors, R. Lahesmaa, and K. V. Rao. Capturing cell-fate decisions from the molecular signatures of a receptor-dependent signaling response. $\mathrm{Mol}$. Syst. Biol. 3:150, 2007.

${ }^{44}$ Kumar, N., A. Wolf-Yadlin, F. M. White, and D. A. Lauffenburger. Modeling HER2 effects on cell behavior from mass spectrometry phosphotyrosine data. PLoS Comput. Biol. 3:e4, 2007.

${ }^{45}$ Lee, M. Y., R. A. Kumar, S. M. Sukumaran, M. G. Hogg, D. S. Clark, and J. S. Dordick. Three-dimensional cellular microarray for high-throughput toxicology assays. Proc. Natl. Acad. Sci. USA 105:59-63, 2008.

${ }^{46}$ Loo, L. H., L. F. Wu, and S. J. Altschuler. Image-based multivariate profiling of drug responses from single cells. Nat. Methods 4:445-453, 2007.

${ }^{47}$ Miller-Jensen, K., K. A. Janes, J. S. Brugge, and D. A. Lauffenburger. Common effector processing mediates cellspecific responses to stimuli. Nature 448:604-608, 2007.

${ }^{48}$ Morgan, E., R. Varro, H. Sepulveda, J. A. Ember, J. Apgar, J. Wilson, L. Lowe, R. Chen, L. Shivraj, A. Agadir, R. Campos, D. Ernst, and A. Gaur. Cytometric bead array: a multiplexed assay platform with applications in various areas of biology. Clin. Immunol. 110:252-266, 2004.

${ }^{49}$ Muschler, G. F., C. Nakamoto, and L. G. Griffith. Engineering principles of clinical cell-based tissue engineering. J. Bone Joint Surg. Am. 86-A:1541-1558, 2004.

${ }^{50}$ Nielsen, J., and S. Oliver. The next wave in metabolome analysis. Trends Biotechnol. 23:544-546, 2005.

${ }^{51}$ Odom, D. T., R. D. Dowell, E. S. Jacobsen, W. Gordon, T. W. Danford, K. D. MacIsaac, P. A. Rolfe, C. M. Conboy, D. K. Gifford, and E. Fraenkel. Tissue-specific transcriptional regulation has diverged significantly between human and mouse. Nat. Genet. 39:730-732, 2007.

${ }^{52}$ Otsuka, H., A. Hirano, Y. Nagasaki, T. Okano, Y. Horiike, and K. Kataoka. Two-dimensional multiarray formation of hepatocyte spheroids on a microfabricated PEG-brush surface. ChemBioChem 5:850-855, 2004.

${ }^{53}$ Paszek, M. J., N. Zahir, K. R. Johnson, J. N. Lakins, G. I. Rozenberg, A. Gefen, C. A. Reinhart-King, S. S. Margulies, M. Dembo, D. Boettiger, D. A. Hammer, and V. M. Weaver. Tensional homeostasis and the malignant phenotype. Cancer Cell. 8:241-254, 2005.

${ }^{54}$ Perez, O. D., and G. P. Nolan. Simultaneous measurement of multiple active kinase states using polychromatic flow cytometry. Nat. Biotechnol. 20:155-162, 2002.
${ }^{55}$ Prudhomme, W., G. Q. Daley, P. Zandstra, and D. A. Lauffenburger. Multivariate proteomic analysis of murine embryonic stem cell self-renewal versus differentiation signaling. Proc. Natl. Acad. Sci. USA 101:2900-2905, 2004.

${ }^{56}$ Rangarajan, A., S. J. Hong, A. Gifford, and R. A. Weinberg. Species- and cell type-specific requirements for cellular transformation. Cancer Cell. 6:171-183, 2004.

${ }^{57}$ Sachs, K., O. Perez, D. Pe'er, D. A. Lauffenburger, and G. P. Nolan. Causal protein-signaling networks derived from multiparameter single-cell data. Science 308:523-529, 2005.

${ }^{58}$ Sevecka, M., and G. MacBeath. State-based discovery: a multidimensional screen for small-molecule modulators of EGF signaling. Nat. Methods 3:825-831, 2006.

${ }^{59}$ Shults, M. D., K. A. Janes, D. A. Lauffenburger, and B. Imperiali. A multiplexed homogeneous fluorescence-based assay for protein kinase activity in cell lysates. Nat. Methods 2:277-283, 2005.

${ }^{60}$ Sivaraman, A., J. K. Leach, S. Townsend, T. Iida, B. J. Hogan, D. B. Stolz, R. Fry, L. D. Samson, S. R. Tannenbaum, and L. G. Griffith. A microscale in vitro physiological model of the liver: predictive screens for drug metabolism and enzyme induction. Curr. Drug Metab. 6:569-591, 2005.

${ }^{61}$ Sodunke, T. R., K. K. Turner, S. A. Caldwell, K. W. McBride, M. J. Reginato, and H. M. Noh. Micropatterns of Matrigel for three-dimensional epithelial cultures. Biomaterials 28:4006-4016, 2007.

${ }^{62}$ Soen, Y., A. Mori, T. D. Palmer, and P. O. Brown. Exploring the regulation of human neural precursor cell differentiation using arrays of signaling microenvironments. Mol. Syst. Biol. 2:37, 2006.

${ }^{63}$ Stachowiak, A. N., and D. J. Irvine. Inverse opal hydrogelcollagen composite scaffolds as a supportive microenvironment for immune cell migration. J. Biomed. Mater. Res. $A$, 2007. In press, doi:10.1002/jbm.a.31661.

${ }^{64}$ Takikita, M., J. Y. Chung, and S. M. Hewitt. Tissue microarrays enabling high-throughput molecular pathology. Curr. Opin. Biotechnol. 18:318-325, 2007.

${ }^{65}$ Underhill, G. H., and S. N. Bhatia. High-throughput analysis of signals regulating stem cell fate and function. Curr. Opin. Chem. Biol. 11:357-366, 2007.

${ }^{66}$ Vargo-Gogola, T., and J. M. Rosen. Modelling breast cancer: one size does not fit all. Nat. Rev. Cancer 7:659-672, 2007.

${ }^{67}$ Wolf-Yadlin, A., N. Kumar, Y. Zhang, S. Hautaniemi, M. Zaman, H. D. Kim, V. Grantcharova, D. A. Lauffenburger, and F. M. White. Effects of HER2 overexpression on cell signaling networks governing proliferation and migration. Mol. Syst. Biol. 2:54, 2006.

${ }^{68}$ Woolf, P. J., W. Prudhomme, L. Daheron, G. Q. Daley, and D. A. Lauffenburger. Bayesian analysis of signaling networks governing embryonic stem cell fate decisions. Bioinformatics 21:741-753, 2005.

${ }^{69}$ Zahir, N., and V. M. Weaver. Death in the third dimension: apoptosis regulation and tissue architecture. Curr. Opin. Genet. Dev. 14:71-80, 2004.

${ }^{70}$ Zaman, M. H., L. M. Trapani, A. L. Sieminski, D. Mackellar, H. Gong, R. D. Kamm, A. Wells, D. A. Lauffenburger, and P. Matsudaira. Migration of tumor cells in $3 \mathrm{D}$ matrices is governed by matrix stiffness along with cell-matrix adhesion and proteolysis. Proc. Natl. Acad. Sci. USA 103:10889-10894, 2006.

${ }^{71}$ Zhang, Y., A. Wolf-Yadlin, and F. M. White. Quantitative proteomic analysis of phosphotyrosine-mediated cellular signaling networks. Methods Mol. Biol. 359:203-212, 2007. 